\title{
Investigation of Changes in Flow Parameters along the Transport Line in Installations for Pneumatic Transport of Cotton
}

\author{
Mahfuza Gapparova1, Bahrom Mirzaev², Mutalov Muhammadodil ${ }^{3}$, Olimjon Sarimsakov ${ }^{3}$ \\ ${ }^{1}$ Tashkent Institute of Textile and Light Industry, Namangan, Uzbekistan \\ ${ }^{2}$ Fergana Polytechnic Institute, Namangan, Uzbekistan \\ ${ }^{3}$ Namangan Institute of Engineering and Technology, Namangan, Uzbekistan \\ Email: olim2083@gmail.com
}

How to cite this paper: Gapparova, M., Mirzaev, B., Muhammadodil, M. and Sarimsakov, O. (2022) Investigation of Changes in Flow Parameters along the Transport Line in Installations for Pneumatic Transport of Cotton. Engineering, 14, 76-83. https://doi.org/10.4236/eng.2022.141007

Received: October 24, 2021

Accepted: January 25, 2022

Published: January 28, 2022

Copyright $\odot 2022$ by author(s) and Scientific Research Publishing Inc. This work is licensed under the Creative Commons Attribution International License (CC BY 4.0).

http://creativecommons.org/licenses/by/4.0/

\begin{abstract}
The article presents the results of a study of changes in the parameters of the flow, velocity and pressure of air along the cotton transportation line in pneumatic transport installations and their dependence on the parameters of the pipeline. The purpose of the research is to theoretically substantiate the choice of the pipeline diameter depending on the properties of the material (cotton), the required processing capacity and pipeline throughput. In the research, an analytical method was used to study changes in the pressure of the air flow along the transportation line at different pipeline diameters. It is established that, according to the existing calculation method, the flow pressure along the transportation line is reduced to zero. At the same time, various scientists have proposed various analytical and empirical dependencies, which, in turn, give different ideas about the resistance of the inner surface of the pipes to the movement of air and material flow and do not describe the real situation, especially when transporting material from a long distance. This requires in-depth theoretical and applied research to establish real patterns of changes in flow parameters along the material (cotton) transportation line.
\end{abstract}

\section{Keywords}

Cotton, Pneumatic Transport Installations, Flow Parameters, Static, Dynamic and Total Pressure

\section{Introduction}

For the transportation of cotton inside cotton processing plants, pneumatic transport units of the suction type are often used. Inside the system, the excess 
pressure is created by the fan, near which the air pressure has the maximum value. As you move away from the fan, both in the direction of suction and discharge, the air speed and pressure decrease. Because of this, the air velocity and pressure along the length of the pipeline are distributed unevenly. This is often explained by the loss of speed and pressure in the pipelines [1] [2] [3] [4].

The connection or removal of a part of the pipeline or the connection or removal of elements of the pneumatic transmission system-elbows, heavy impurity catcher (revisions), separator, diffusers and confusers is accompanied by a decrease or increase in pressure inside the pipeline. In order to understand the essence of this phenomenon, it is necessary to study the process of pressure occurrence.

Any aerodynamic installation contains at least 3 components. This is the fan and connected to it, the suitable and outgoing pipelines. In any design, the pressure of the fan creates its impeller, which shovels air particles from one side to the other, which creates a moving flow of air inside the housing, which has an inlet and outlet windows, which are connected respectively to the inlet and exhaust pipes. During rotation, the impellers (or wheels) cut off the surrounding air layers from the entrance window and throw them towards the exit window. Moreover, a sparse medium is formed in the input window, and a compacted medium is formed at the output.

The discharged medium is a vacuum, which attracts nearby particles, i.e., particles of air and creating a corridor for the movement, formed a stream that is part of the fan. Similarly, the repulsive force of the compacted environment also forms a flow, only the exhaust. Fan binds these threads into one, i.e., during operation, the fan sucks air flow or Aero mixture on one side and outputs it to the other side. According to this, the indices of the incoming and outgoing flow are the same. Rate of discharge (also called compaction), in fact, is the indicator measured in units of force (or mass) per unit volume (this is the unit of density). However, to date for no science of such a measure as an indicator of low (or compaction), there is no means of measurement. But, there is the concept of pressure, which is measured by the magnitude of the force per unit area.

\section{Mathematical Description of Changes in Flow Parameters along the Transportation Line}

This, the power that is in the field of vacuum pulling pipe wall to the center of the stream, and in the field of sealing pressure on pipe wall from the inside. And this power can be measured instrumental and attributing its importance to the area of action of this force determines the pressure value. In pipelines, the forces acting on the pipe wall perpendicular to cause static pressure $P_{s t}$ the forces acting along the pipeline-dynamic $P_{d .}$. Total pressure $P_{\mathrm{n}}$ is the algebraic sum of the absolute values of $P_{\text {д }}$ the dynamic and static $P_{\text {st }}$ pressure [5] [6] [7] [8]:

$$
P_{s t}=\rho g h,
$$




$$
\begin{gathered}
P_{d}=0.5 \rho v^{2} \\
P_{p}=/ P_{s t} /+/ P_{d} /
\end{gathered}
$$

where $\rho$-is the density $\left(\mathrm{kg} / \mathrm{m}^{3}\right), v$-speed $(\mathrm{m} / \mathrm{s})$ of flow; $g$-gravitational acceleration, $\mathrm{m} / \mathrm{s}^{2} ; h$-height photometrically pressure, which is equal to the height of air column or liquid, giving a pressure to the $P_{C T}$ on the contact surface equal to $1 \mathrm{~m}^{2}$.

It is known that the dynamic pressure depends on density and air velocity, in the absence of vacuum (or exhaust) gas through the wall of the pipe (or pipe joints), i.e. the constancy of the speed of air, it does not change along the entire length of the pipeline and its possible to define the flow-rate measurement. And static pressure, assuming the density of known value that depends only on the height of the column of air, which is not determined instrumentally. This pressure is estimated a direct measurement at the beginning (the mouth) and the end (of the fan) of the pipeline. Judging by the formula, the static pressure depends on the height of the column of gas, which by its weight presses on the support surface, and this in turn is the distance that starts from the point where the pressure is minimum (or zero) value and continues to the point of contact pressure force with a supporting surface where it has maximum value. At a constant cross-section and resistance to duct static pressure from the mouth of the duct to the fan varies in a linear relationship [9]-[14].

Imagine that the origin POL is located at the fan $0 \mathrm{~L}$-axis represents the length of the pipeline and $0 \mathrm{P}$-static pressure. For this case the equation of the line can be described as:

$$
\left(P-P_{u}\right) /\left(P_{b}-P_{u}\right)=\left(L-L_{0}\right) /\left(L_{b}-L_{0}\right),
$$

where, $P_{H}$ is the initial or nominal pressure (i.e., pressure of the fan, which is equal to the certified value of the pressure generated by the fan) $\mathrm{Pa} ; P_{b}-$ the final pressure, or pressure at the mouth of the pipeline, $\mathrm{Pa} ; L_{0}$ and $L_{b}$, respectively, the initial and final length of pipeline, $\mathrm{m}$. Imagine that the countdown starts right at the fan and take $L=L_{0}=0$, then the mouth of the pipe $L=L_{b}$. If, given these conditions, to solve the equation for $\mathrm{R}$, we get:

$$
P=\left(P_{b} L+P_{H}\left(L_{b}-L\right)\right) / L_{b},
$$

Check: if $L=0, P=P_{H}$, and when $L=L_{b}, P=P_{b}$. Hence, the equation correctly describes the law of variation of pressure along the transport line.

On the other hand, at the mouth of the pipeline pressure decreases to a pressure value of $P_{K}$ is required to overcome the resistance of the pipeline:

$$
P_{\kappa}=0.5 \rho v^{2} \lambda L_{b} / d,
$$

where, $\lambda$ is the drag coefficient. Accordingly, the pressure at the mouth of the pipeline will be equal to:

$$
P_{b}=P_{H}-0.5 \rho v^{2} \lambda L_{b} / d .
$$

If the equation to describe the relative static pressure, the pressure at any 
point on pneumotrack will be equal to:

$$
P_{c m}=P_{\mu c}-0.5 \rho v^{2} \lambda L / d,
$$

Here $P_{H C}$ is the nominal static pressure $\mathrm{Pa}$. And total pressure $P_{p}$ according to (2), (3) and (8):

$$
P_{p}=P_{\mu p}-0.5 \rho v^{2}(\lambda L / d-1),
$$

Here, $P_{\mu p}-$ full rated pressure of the passport is also the index fan $\mathrm{Pa}$.

Internal diameter of the pipeline is equal to $d=0.315 ; 0.355 ; 0.4 \mathrm{~m}$, the air density $\rho=1.2 \mathrm{~kg} / \mathrm{m}^{3}$. The resistance coefficient for clean air for rough pipes is often determined by the formula of Shiferson [1] [2] [15] [16]:

$$
\lambda=0.111(a / d)^{0.25},
$$

when $a=0.133$

$$
\begin{aligned}
& d=0.315 \mathrm{~mm}-\lambda=0.089 ; \\
& d=0.355 \mathrm{~mm}-\lambda=0.087 ; \\
& d=0.4 \mathrm{~mm}-\lambda=0.084 ;
\end{aligned}
$$

when $a=0.044$

$$
\begin{aligned}
& d=0.315 \mathrm{~mm}-\lambda=0.068 ; \\
& d=0.355 \mathrm{~mm}-\lambda=0.066 ; \\
& d=0.4 \mathrm{~mm}-\lambda=0.064 .
\end{aligned}
$$

Here $a$-coefficient of roughness of the pipe, which for the new pipes is equal to $a=0.133$; used for pipes $a=0.044 \mathrm{~mm}$. With this in mind, for different pipe diameters.

Analysis of Equation (9), conducted on a computer with different flow parameters are presented in Figure 1.

Photos 1, 2 and 3-full line pressure, 4, 5 and 6 lines of static pressure and dynamic pressure line 7. At the same time, 1 and $4-400 \mathrm{~mm}$ pipe, 2 and $5-355$ $\mathrm{mm}$ pipe, 3 and 6 - $315 \mathrm{~mm}$ pipe.

The results show that the dynamic pressure under the condition of complete tightness of the system has a constant value along the line of current. And static and total pressure linearly decreases from the point of disturbance, i.e., from the

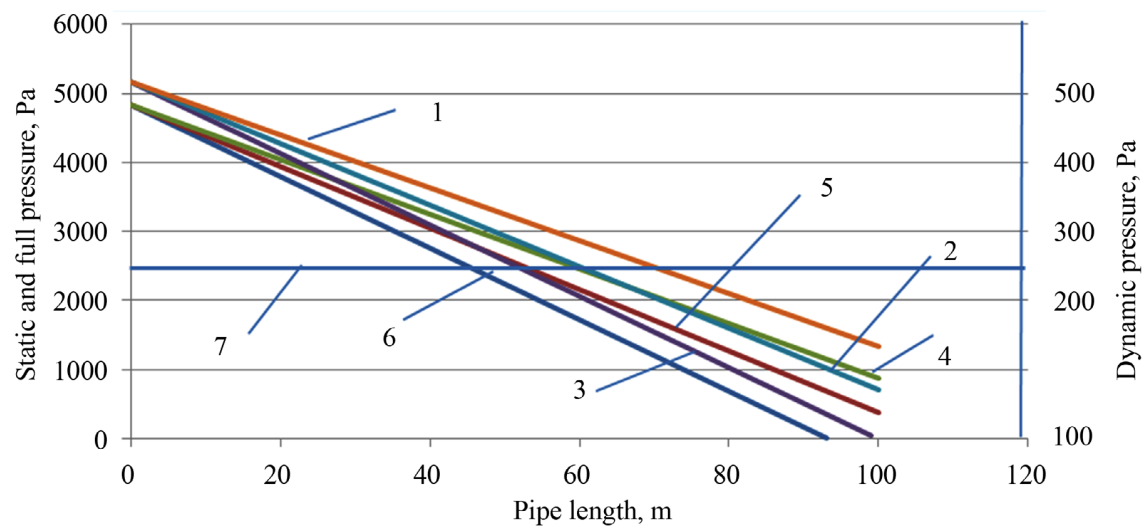

Figure 1. Distribution of statically and dynamic pressure along the line of the pipe. 
fan to the extremities of pneumothrax. The figure shows that at smaller diameters the decrease in pressure more intense. This shows high resistance of pipes of a smaller size relatively large. For example, if pipe diameter $315 \mathrm{~mm}$ static and dynamic pressure approaching zero at a distance of 95 to $100 \mathrm{~m}$. Important this time graphics of pressure when the diameter of $355 \mathrm{~mm}$ (2-line) and $400 \mathrm{~mm}$ (4-line) intersect and the values are very close to each other. This shows that replacement of the pipeline diameter $400 \mathrm{~mm}$ pipeline diameter $355 \mathrm{~mm}$ to large losses of pressure and power will not. According to this, the pipe diameter is 355 $\mathrm{mm}$ can successfully replace the pipes of $400 \mathrm{~mm}$ diameter.

Indeed, many studies report an increase in the resistance of the pipeline with decreasing diameter of the pipeline. However, by Blasius [1] [2] found that for hydraulically smooth tubes the increase in air velocity at constant diameter of the pipe reduces the resistance of the pipeline. They have the inversely proportional dependence of drag coefficient on Reynolds number:

$$
\lambda=0.3164 / \mathrm{Re}^{0.25},
$$

In turn, the Reynolds number is defined as the product of duct diameter and air velocity:

$$
\operatorname{Re}=\rho d v / \mu .
$$

Here $\mu$ - the coefficient of dynamic viscosity of air $\left(1.85 \times 10^{-5} \mathrm{~Pa} \cdot \mathrm{s}\right)$.

\section{Analysis of Research Results}

The results of the analysis of (13) are presented in Table 1.

In the table, $\lambda_{b}$ is the drag coefficient for the Blasius; $\lambda_{e}$ is the experimental drag coefficient. If you pay attention to the results of the analysis can be seen that the decrease in pipe diameter leads to an increase in resistance and increase in flow velocity to decrease. In addition, the experimental results under odds on Shiferson, more of the coefficients for the Blasius. In this case, the experimental drag coefficient $\lambda_{e}$ are determined according to the equation:

$$
\lambda=\left(0.5 \rho v^{2}+P_{u}-P_{n}\right) d /\left(0.5 L \rho v^{2}\right)
$$

the substitution of actual values of pressure of air at the mouth of the pipeline $(\mathrm{RP})$ and the fan pneumatic conveying installation $\left(P_{H}\right)$.

Table 1. The dependence of the aerodynamic drag from air velocity and duct diameter.

\begin{tabular}{ccccccc}
\hline & \multicolumn{5}{c}{$\lambda$ at different air velocities } \\
\cline { 2 - 7 }$D, \mathrm{~m}$ & \multicolumn{2}{c}{$v=15 \mathrm{~m} / \mathrm{s}$} & \multicolumn{2}{c}{$v=20 \mathrm{~m} / \mathrm{s}$} & \multicolumn{2}{c}{$v=25 \mathrm{~m} / \mathrm{s}$} \\
\cline { 2 - 7 } & $\lambda_{b}$ & $\lambda_{e}$ & $\lambda_{b}$ & $\lambda_{e}$ & $\lambda_{b}$ & $\lambda_{e}$ \\
\hline 0.400 & 0.0127 & 0.022 & 0.0118 & 0.0206 & 0.007 & 0.019 \\
0.355 & 0.0130 & 0.023 & 0.0120 & 0.0216 & 0.0115 & 0.020 \\
0.315 & 0.0135 & 0.024 & 0.0125 & 0.022 & 0.0118 & 0.0216 \\
\hline
\end{tabular}


From the results of the analysis, it can be concluded that, indeed, an increase in the resistance of pipes of smaller diameter can be compensated by an increase in flow [3]. This shows the possibility of successful use of pipes with a diameter of 355 and $315 \mathrm{~mm}$ in the installation of pneumatic transport under pressure for raw cotton [17] [18] [19] [20] [21].

At the same time, it should be particularly noted that, according to the current calculation method, the flow pressure along the transport line is reduced to zero. At the same time, different scientists have proposed different analytical and empirical dependencies, which, in turn, express different opinions about the resistance of the inner surface of pipes to the movement of air and material flows and do not describe the real situation, especially when transporting material over long distances. In our opinion, the established patterns and values are correct for the conditions and length of the pipeline under which these studies were conducted, about which the authors do not provide specific information. But, in any case, a common feature of all such studies is that with an increase in the distance of transportation, i.e. the length of the pipeline to a certain value, the air pressure decreases to zero. However, our applied research has not confirmed this statement, and under any conditions when there is pressure at the beginning of the pipeline, i.e., at a pressure source (for example, at a fan), if the cross-section of the pipeline is passable, the air pressure does not decrease to zero. This requires deeper theoretical and applied research to determine the actual change in flow parameters along the material (cotton) transportation line, which is the goal of our further research.

\section{Conclusions}

1) Theoretical studies have shown and proved a decrease in static and total pressure along the transportation line, according to a linear pattern.

2) It is established that the air pressure varies depending on the diameter and aerodynamic resistance of the pipeline and the distance of the transport highway.

3) It is established that the existing analytical and empirical dependencies do not describe the real situation, especially when transporting material from a long distance, which requires in-depth theoretical and applied studies of changes in flow parameters along the material transportation line.

\section{Conflicts of Interest}

The authors declare no conflicts of interest regarding the publication of this paper.

\section{References}

[1] Altschul, A. (1987) Hydraulics and Aerodynamics. Stroyizdat, Moscow.

[2] Loytsyansk, L. (2003) Liquid and Gas Mechanics. Drofa, Moscow.

[3] Obidov, A.A. and Sultanov, M.M. (2020) To Research the Method of Separating Fi- 
bers Suitable for Spinning on a Needle Drum. International Scientific and Practical Conference Cutting Edge-Science, Shawnee, 29-30 June 2020, 128-131.

[4] Sultonov, M.M. and Obidov, A.A. (2020) Study of Fiber Motion in a Needle Drum Fiber Separation Device. Universum: Technical Science, 5-74, 33-37.

[5] Sultanov, M. and Obidov, A. (2020) Investigation of Working Parts of Fixed Device Designed to Separate Spinning Fibers from Fibrous Waste That Can Be Spun. Journal of Critical Reviews, 7, 2314-2322.

[6] Obidov, A.A. and Sultanov, M.M. (2020) Study of Technological Parameters of Fiber Separation Device. International Journal of Psychosocial Rehabilitation, 24, 6400-6407. https://doi.org/10.37200/IJPR/V24I5/PR2020624

[7] Azamatovich, B.U.O.A., Juraboyevich, M.K. and Mirzarahmatovich S.M. (2019) Research Capacity of the Fiber of Long Fiber Separating Drums from Waste Fibers Composition. International Journal of Innovative Studies in Sciences and Engineering Technology, 5, 28-31.

[8] Obidov, A., Mamatqulov, O. and Sultanov, M. (2018) Theoretical Analysis of the Movement of Cotton Piece on the Slope Surface. International Conference Science and Practice: A New Level of Integration in the Modern World, Berlin, Vol. 82, 151-156.

[9] Axmedxodjayev, K., Umarov, A. and Ortiqova, K. (2019) Investigation of the Ginning Process on ДП Series Saw Gin Stands. Engineering, 11, Article ID: 94702. https://doi.org/10.4236/eng.2019.118036

[10] Ergashev, J., Kayumov, J., Ismatullaev, N. and Parpiev, U. (2020) Theoretical Basis for Calculating the Determination of the Optimal Angle of Rotation of the Slit and Air Velocity. International Journal of Advanced Science and Technology, 29, 12776-12784.

[11] Obidov, A.A. (2006) Investigation of the Working Surface of a New Seeding Machine. Journal of Textile Problems, 2, 29-32.

[12] Obidov, A., Vokhidov, M. and Abdurahmonov, J. (2021) Exploring the Efficiency of Experimental Construction of Sorting Ginned Cotton Seed Machine. Engineering, 13, 18-29. https://doi.org/10.4236/eng.2021.131002

[13] Obidov, A.A. (2007) Improvement of the Technology of Cleaning and Sorting of Ginned Seeds. Dissertation of Candidate of Technical Sciences, TITLI, Tashkent, $200 \mathrm{p}$.

[14] Mukhitdinov, A.M. and Makhkamov, R.G. (1989) The Load on the Teeth of the Comb Drum Set of the Rotary Spinning Machine. Dep. Article, Namangan.

[15] Kholmirzaev, F., Azimov, S., Abdurahimov, K. and Sarimsakov, O. (2019) Investigation of the Loss of Air Pressure in Pipeline of the Sotton Pneumatic Conveying. Saudi Journal of Engineering and Technology, 4, 23-27.

[16] Sarimsakov, O. (2016) The Possibility of Reducing Cotton Consumption in Cotton. American Journal of Science and Technology, 4, 68-72.

[17] Sarimsakov, O. and Gaybnazarov, E. (2016) About Energy Consumption in Pneumatic Conveying of Raw Cotton. American Journal of Energy and Power Engineering, 3, 26-29.

[18] Sarimsakov, O., Khusanov, C. and Muradov, R. (2016) The Change of Air Pressure Along the Pipeline Installation for Pneumatic Conveying Raw Cotton. Journal of Engineering and Technology, 3, 89-92.

[19] Abdukarimovich, M.O., Ibragimovich, A.K. and Sharipjanovich, S.O. (2018) Cylinder for Pneumomechanical Spinning Machines. Engineering, 10, 345-356.

https://doi.org/10.4236/eng.2018.106025 
[20] Tursunov, I., Rajapova, N., Sarimsakov, O. and Mardonov, B. (2019) The Movement of a Mixture of Cotton with an Air Stream during Pneumatic Transport by Pipeline of Variable Cross Section. Engineering, 11, 531-540.

https://doi.org/10.4236/eng.2019.118037

[21] Fathollahi, M., Ahmadi, M.E. and Hosseini, S.M.J. (2019) Electrostatic Charge Generation in Pneumatic Conveying Process: Effect of Particle Properties. Journal of Power and Energy Engineering, 7, 1-11. https://doi.org/10.4236/jpee.2019.76001 